\title{
Direct Determination of Polycyclic Aromatic Hydrocarbons in Solid Matrices Using Laser Desorption/Laser Photoionization Ion Trap Mass Spectrometry
}

\author{
A. A. Specht and M. W. Blades \\ Department of Chemistry, University of British Columbia, Vancouver, British Columbia, Canada
}

\begin{abstract}
The development and characterization of a new instrument for solid sampling which couples IR laser desorption followed by UV laser photo-ionization and analysis using an ion trap mass spectrometer has been investigated. For calibration, a new type of solid sample preparation involving activated charcoal as the solid substrate was used. This solid sample provided a steady signal for several thousand laser shots, which allowed optimization of the experimental procedure. It was found that both the IR and UV intensity and the delay between them play an important role in both the magnitude and type of signals observed. A method of gas phase accumulation with multiple laser shots was examined. Finally, this technique was demonstrated to be effective in providing direct qualitative information for N.I.S.T. SRM 1944 river sediment sample with no sample pre-treatment. (J Am Soc Mass Spectrom 2003, 14, 562-570) (c) 2003 American Society for Mass Spectrometry
\end{abstract}

$I^{2}$ on trap mass spectrometers are one of the most common and powerful mass analyzers in use because they have a relatively high scan speed, are sensitive, and are inherently capable of performing $\mathrm{MS}^{\mathrm{n}}$ experiments [1-3]. Also, ion traps are well suited for pulsed laser experiments such as MALDI because, as is the case with time-of-flight (TOF) instruments, a mass spectrum of the entire sample can be obtained from a single laser shot. However, ion traps suffer from two disadvantages that limit their potential effectiveness. First, the total number of ions that may be trapped simultaneously can be limited by space charge. This phenomenon, caused by coulombic repulsion of similarly charged ions, results in peak broadening and mass shifts in the spectrum. The second limitation concerns the trapping efficiency of externally introduced ions. Externally introduced ions must penetrate an alternating electric field and then undergo sufficient collisions with the background buffer gas in order to be cooled and efficiently trapped. The trapping efficiency of externally created ions depends on a variety of factors including; the initial kinetic energy of the injected ions, the phase angle of the radio frequency (RF) field at the time at which the ions enter the trap, and the amplitude of the RF voltage $\left(q_{z}\right)[4]$. A variety of theoretical studies have suggested that the trapping efficiency of externally

Published online April 24, 2003

Address reprint requests to Dr. M. W. Blades, Department of Chemistry, University of British Columbia, 2036 Main Hall, Vancouver, British Columbia V6T 1Z1, Canada. E-mail: blades@chem.ubc.ca injected ions is anywhere between 5\% [5] for normal RF power supplies to $100 \%$ [6] for fast switching, sudden onset, RF power supplies. Using the two-laser sampling/ionization approach described in this paper, these two effects are minimized because analyte ions are formed selectively at the center of the trap. In addition, the two-laser sampling approach is inherently an excellent method for the direct analysis of solid samples and extends the already wide capabilities of the ion trap.

The two-laser method for solid sampling has been known for some time. It was first developed for mass spectrometry of involatile and/or thermally labile organics in the mid-1980s [7-9]. Since that time it has been further developed and used for a variety of solid samples including polymers [10], soot and coal tar [11], dyes [12], and molecular adsorbates [13]. Two-laser mass spectrometry, as it has been previously applied, is a three-step process. The first step involves using a pulsed IR laser (typically a $\mathrm{CO}_{2}$ laser) for thermal desorption of the sample. If the wavelength and power density are chosen correctly (typically around $10^{6}$ $\mathrm{W} / \mathrm{cm}^{2}$ ) thermal desorption will produce a plume of intact neutrals with very little ionization or sample decomposition [14]. The second step in this process involves the use of a pulsed UV laser (typically a $\mathrm{Nd}$ :YAG laser), to ionize the analyte present in the neutral desorbate plume. Again, if the wavelength and power are chosen correctly, the ionization process can be extremely gentle and produce mainly intact molecular ions [15]. Finally, in virtually every reported exam- 
ple of the use of this technique, the ions are detected using a time of flight mass spectrometer $[8,12,16]$. It is in the temporal and spatial separation of the desorption and ionization processes where the advantages of the technique are gained. The separation allows an independent optimization of both steps thus making the process extremely gentle and sensitive.

The mode of ionization in almost every two-laser experiment involves resonance enhanced multiphoton ionization (REMPI). The ionization potential for many organics is on the order of 7-13 eV, therefore, single photon ionization would require a wavelength in the vacuum UV. In contrast, for the REMPI experiment, ionization relies on the molecule absorbing two photons. This mode of operation provides a high level of selectivity in the ionization process because the ionization efficiency is dramatically improved if the wavelength of the laser matches an electronic transition in the molecule. An improved ionization efficiency results because a real electronic excited state will persist longer than a "non-real" virtual excited state. This longer-lived excited state will therefore have a higher probability of absorbing a second photon to induce ionization. The optical selectivity afforded by this process can therefore allow preferential ionization of analyte molecules over matrix species that do not have an electronic absorption at the REMPI wavelength. This result is critical if one hopes to achieve good sensitivity in the presence of real matrix. The two-laser technique has been shown to have very low detection levels; for example, attomolar detection limits have been published for the analysis of aniline adsorbed on silica [17]. Finally, because the second photon often induces ionization with little excess energy, a relatively small amount of molecular fragmentation will result compared to a direct laser desorption experiment, thus simplifying the spectra.

There are several advantages associated with the two-laser method over more traditional laser desorption techniques for use with an ion trap. The selectivity of the ionization step can allow one to directly assay real samples, minimizing concern that the background matrix would induce space charge problems because only molecules of interest are ionized. Secondly, the possibility exists of using stored waveform inverse fourier transform (SWIFT) ion isolation coupled with multiple laser shots in order to sample the result of several desorption/ionization steps, resulting in increased sensitivity. Perhaps the greatest advantage, however, is realized when one considers that the ionization step can take place in the center of the ion trap, where trapping efficiency is highest. This should, in theory, allow a greater level of sensitivity when compared with a MALDI experiment in which externally generated ions are injected. Hanley and co-workers have already published one example of performing two-laser MS in an ion trap [13, 18]. Hanley's work differs from that presented here in both purpose and methods; the goal of his group's work was focused on surface analysis, while this work was done with the intent of assaying the content of "real" samples directly. Consequently, the geometry, design, and implementation of the two instruments are somewhat different.

The model compounds chosen to investigate the feasibility of this methodology are a group of polycyclic aromatic hydrocarbons (PAHs). The PAHs represent a large family of molecules which are produced via both natural and anthropogenic processes and are found ubiquitously throughout the environment $[19,20]$. Currently there are several methods available for the analysis of PAHs in solid matrices. Usually these methods involve some form of extraction from the matrix, typically using a Soxhlet Extractor, followed by separation via chromatography [usually gas chromatography (GC)] followed by detection using mass spectrometry MS [21,22]. Other separation and detection techniques have been suggested, but none have reached the level of routine analysis [23-25]. Another important consideration in the field of PAH analyses of environmental samples is the issue of sample preparation. Typically, most forms of $\mathrm{PAH}$ analysis require that the samples are cleaned and the PAHs removed from the solid matrix. This process is neither inexpensive nor fast, and certainly not trivial because one solid sample often contains PAHs in a variety of physico-chemical states [26]. Once the PAHs have been extracted from their native matrix, the analyst must also be concerned with issues of solubility, storage, biotransformation, and photo-degradation. As a solution to this persistent problem, several groups have attempted direct analysis on the solid matrix. Some of the more successful applications include secondary ion mass spectrometry (SIMS) [27], fast atom bombardment (FAB) [28], and laser desorption (LD) [29]. These techniques, however, are often non-selective in nature and produce complex mass spectra with a variety of peaks resulting from molecular fragmentation of both the analyte and the matrix.

Two-laser mass spectrometry has been applied to PAH analysis successfully by several groups [30, 31]. These groups have performed PAH analysis in both artificial [32] and natural matrices [11, 30]. Perhaps the best-known example of this technique was the examination of Martian meteorites [33, 34]. It should be noted that in all of these examples, the mode of mass spectrometry used was always a time-of-flight. While a TOF is very useful when used in a two-laser experiment, it is not capable of $\mathrm{MS}^{\mathrm{n}}$ - a feature which may prove useful for obtaining structural and possibly isomeric information.

This paper describes the use of two-laser sampling directly within the volume of an ion trap. A set of solid PAH standards were made in order to characterize the instrument and find the optimum conditions for operation. The effect of several parameters on the mass spectrum acquired were examined including the energy of both the IR and UV lasers, the effect of sample concentration on signal, and the delay time between laser shots. The functionality of the ion trap was dem- 


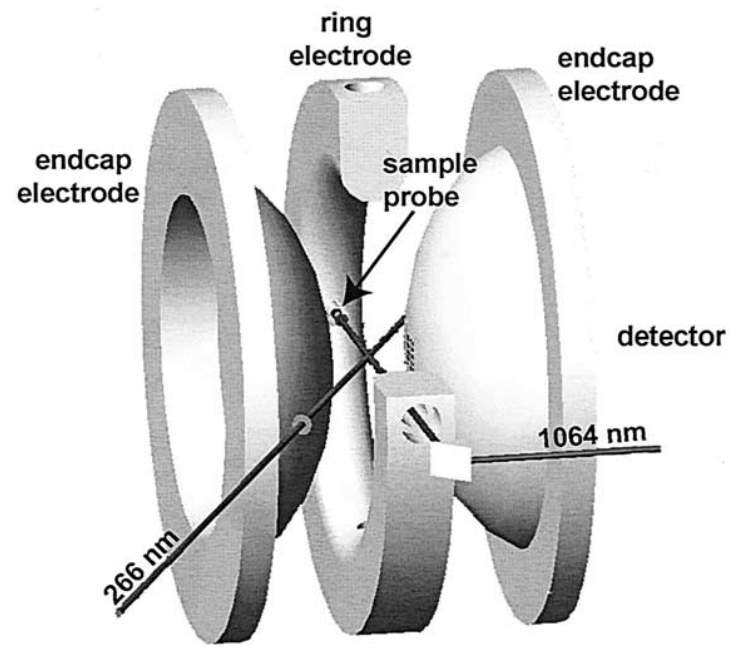

Figure 1. IR laser desorption, UV laser photoionization instrument at U.B.C.

onstrated by allowing the selective accumulation of one analyte over several laser shots. Finally, as a demonstration of the technique's applicability to real samples, a standard reference solid sample containing known PAHs was analyzed.

\section{Experimental}

A diagram of the instrument is shown in Figure 1. The physical ion trap manifold shown was described previously [35], however, extensive modifications have been made to the control and acquisition systems. The iontrap electrodes were home built in the U.B.C. mechanical shop and are of the "ideal quadrupole geometry" (i.e., not stretched) and are enclosed in a vacuum manifold. The vacuum manifold was specifically designed with optical experiments in mind. It has four optical ports located in-line with the asymptotes of the ion trap. This allowed two direct lines of sight diagonally through the ion trap, between the endcaps and the ring electrode. A fifth optical port was placed in-line with the center of the ring electrode. This allowed optical access to two $2.5 \mathrm{~mm}$ holes drilled into the ring electrode opposite each other. Opposite this fifth optical port was a vacuum interlock which was custom-built to allow a sample probe to be inserted into the ion trap flush with the inside of the ring electrode without the need to break the vacuum.

For sample desorption, a Nd:YAG laser (DCR-2A, Spectra Physics Lasers, Mountain View, CA) operating at $1064 \mathrm{~nm}$, with a pulse width of $10 \mathrm{ns,} \mathrm{was} \mathrm{used.} \mathrm{This}$ laser was directed, unfocused, into the ion trap with two quartz prisms (Melles Griot, Rochester, NY) and the energy of the laser was significantly reduced by a series of quartz beam diffusers. This was necessary because the minimum energy for reliable lasing was far greater than that required for desorption. The average laser power measured in the trap at the probe surface was typically $10^{5} \mathrm{~W} / \mathrm{cm}^{2}$. Since the beam diameter was much larger than the $2.5 \mathrm{~mm}$ aperture in the electrodes, it was the aperture size that was used to calculate the power/area ratio. For photo-ionization, a second Nd: YAG laser (Lumonics HY400, 10 ns pulses), frequency quadrupled to provide output at $266 \mathrm{~nm}$, was directed through one of the optical ports along the diagonal between the endcaps and the ring electrode. The laser passed through the center of the ion trap after being slightly focused by a $1000 \mathrm{~mm}$ bi-convex lens (Melles Griot). Typical energy levels for the UV laser were on the order to $40 \mu \mathrm{J} /$ pulse.

In-house written programs operating on a $350 \mathrm{MHz}$ Pentium II computer were used to control the timing and data acquisition for the experiment. Instrument control and data acquisition software was written in the LabView (National Instruments, Austin, TX) programming environment. The digital-to-analog (DAC) and the analog-to-digital (ADC) conversion took place on a PCI board that was also capable of accepting and producing a variety of digital pulses (PCI-MIO-16XE10, National Instruments). A GPIB interface board, the use of which will be described later, (AT-GPIB/TNT National Instruments) was also used. An Extranuclear Laboratories Inc. (Pittsburgh, PA) RF quadrupole power supply (Model 001-1), modified for operation from 0.6 to $3.0 \mathrm{MHz}$, was used. Capacitance matching was achieved using a High-Q Head (Model number 012-16), also from Extranuclear Laboratories. The maximum RF output was approximately $\sim 3000 \mathrm{~V}$ (zero to peak), and the frequency was fixed at $0.967 \mathrm{MHz}$. Following sample desorption and ionization, a 50-1000 ms cooling period using He buffer gas, at a constant RF, was used. After the cooling period, a single frequency waveform was applied to one electrode with a Stanford Research Systems (Palo Alto, CA, model SRS DS345) function generator. Synchronized with this waveform, the RF potential was ramped to generate a mass spectrum of the ions using the resonance ejection mode of operation. The ejected ions were detected using an ETP (Sydney, Australia) model AF138 electron multiplier held at $-1.7 \mathrm{kV}$. The signal from the detector was amplified with a gain of $10^{7} \mathrm{~V} / \mathrm{A}$ using a Kiethly (Cleveland, $\mathrm{OH}$ ) model 428 current amplifier. The ADC on the PCI board then sampled this amplified signal.

A second computer was installed in this system to allow for SWIFT waveforms to be applied to the end caps. This process allowed the selective ejection of ions with motion at the applied frequency. This system was based on a PCI-321 arbitrary function generator (PCI Instruments Inc., Akron, $\mathrm{OH}$ ) card onto which the calculated waveform was loaded. A second computer was used because the large processing overhead required for the SWIFT calculations would slow down the primary computer.

The electronic systems required to provide precise timing for the two lasers, the DAC/ADC and the RF power supply were designed and constructed at U.B.C. by the electronic services group and the authors. The Nd:YAG lasers were designed to operate at $10 \mathrm{~Hz}$, 
however the ion trap electronics could be run only at a maximum of $1 \mathrm{~Hz}$. To compensate for this frequency mismatch, a user built protocol was required. In the simplest case, the flash lamps for both Nd:YAG lasers were linked and allowed to fire at the DCR-2A internal clock rate of $10 \mathrm{~Hz}$. This pulse train was monitored with a "trigger monitor". When a laser pulse was required, a TTL pulse from a Stanford Research Systems (Palo Alto CA, model SRS DG535) digital delay generator sent an "enable" pulse to the "trigger monitor", which when enabled allowed, on receipt of the next flash lamp pulse, a pulse to be fed into a second digital delay generator (model 400, Berkeley Nucleonics Corp., San Rafael, CA) which triggered the Q-switches at two separate programmable delays for each laser. This produced a very stable and reliable delay between the firing of the two lasers. The typical delay between the IR and UV laser pulse was user set to $30 \mu \mathrm{s}$, but with some small modifications to the timing system, the laser delay for UV could easily be varied between -10 to $400 \mu$ s with respect to the IR laser pulse. The jitter in the laser delay was less than $1 \mu \mathrm{s}$. A second advantage of running the lasers this way is that the energy produced by the lasers is much more stable with a constant flashlamp rate compared to random laser firings. The added stability occurs because the Nd:YAG rod temperature plays a role in the beam quality and power output and with a steady rate of flashlamp firing a steadier rod temperature can be maintained thus allowing a smaller shot to shot energy fluctuation.

While the typical power densities and time delays for the lasers were listed above, it should be mentioned that the exact power outputs and the exact time delays were known for each mass scan. This was made possible by installing two fast photodiodes (Melles Griot) each of which was able to detect the arrival of one of the laser pulses. The photodiodes were connected to a $400 \mathrm{MHz}$ digital oscilloscope (model TDS 380, Tektronix, Wilsonville, OR) that recorded both the intensity of the lasers and the time delay between shots. The oscilloscope then reported these results through the GPIB interface to the computer. In this way, information about the laser intensity and the laser delays could be bundled with the mass spectral data for each mass scan for subsequent signal processing.

The eventual goal of this research was to evaluate this system for its potential to assay real environmental solid samples, for example soil and sediments, however, before this could be done, a reliable method of preparing standards was required. The technique for "standard" sample preparation, which was eventually used, is similar to that used by De Vries [36], involved activated charcoal as a solid substrate on which polycyclic aromatic hydrocarbons were adsorbed. These solid standards were made by first preparing a variety of standard solutions of the PAHs in hexane (HPLC grade). All compounds were acquired from SigmaAldrich, (Milwaukee, WI) and were used as received. The six PAHs used in this study were: acenaphthene

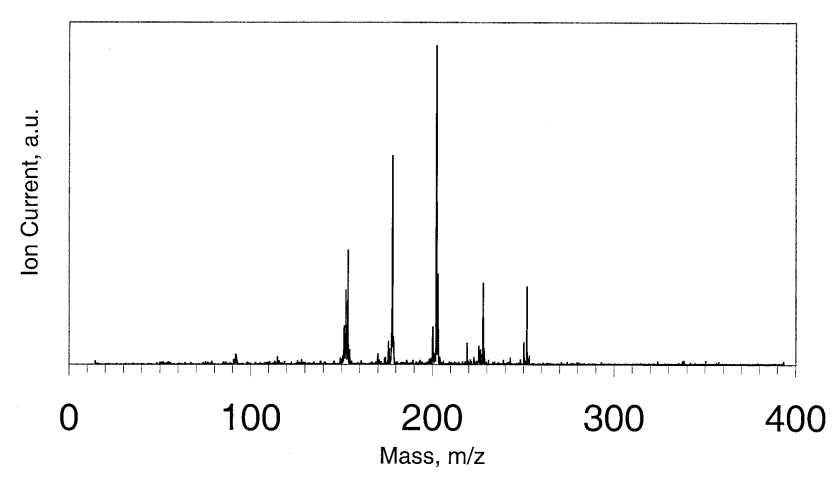

Figure 2. Two-laser mass spectrum of 5 PAHs (acenaphthene$154 \mathrm{Da}$, phenanthrene-178 Da, pyrene-202 Da, chrysene-228 Da, benzo(a)pyrene-252 Da) adsorbed on activated charcoal in the concentration range of $25 \mu$ moles of $\mathrm{PAH} /$ gram of charcoal.

(mass $154 \mathrm{Da})$, phenanthrene (178 Da), pyrene (202Da), chrysene (228 Da), benzo(a)pyrene (252 Da), and coronene (300 Da). These solutions were then pipetted onto previously weighed amounts of the activated charcoal. The charcoal/hexane slurries were then sonicated for 30 min and left overnight for the hexane to evaporate, leaving the PAHs adsorbed onto the activated charcoal. Small amounts, on the order of $1 \mu \mathrm{g}$, of the "standard solid samples" were then placed in a small sampling cup (dimensions $1.5 \mathrm{~mm}$ diameter and $0.8 \mathrm{~mm}$ depth) and mechanically pressed (using a custom-built press, Chemistry Department Mechanical Shop, U.B.C.) into place. The press was designed with mechanical stops that defined the sample press depth thus producing sample pucks which were all of identical physical dimensions $(0.4 \mathrm{~mm}$ thick samples). The pressure required to press the charcoal sample was less than 100 PSI. In addition to an extensive range of standard samples which were tested, a standard reference material (SRM 1944, New York-New Jersey Water Way sediment, U.S. Department of Commerce National Institute of Standards and Technology, Gaithersburg, Maryland), certified to contain a variety of PAHs was also examined.

\section{Results and Discussion}

\section{Standard Sample Characterization}

Several hundred different charcoal/PAH standards were examined using the sample preparation procedure described above. A typical example of the mass spectrum obtained for one of these samples, containing five PAHs at concentrations of approximately $25 \mu$ mole $\mathrm{PAH} /$ gram of charcoal is shown in Figure 2. Note that the spectrum is relatively "clean" in that it shows very few peaks other than for the five PAHs. It should also be noted that very little fragmentation was observed in any of the trials performed. This result is consistent with those seen previously in the literature for both PAHs [11, 30-32] and other large molecules [9, 12, 18, 37] with the two-laser set-up. 


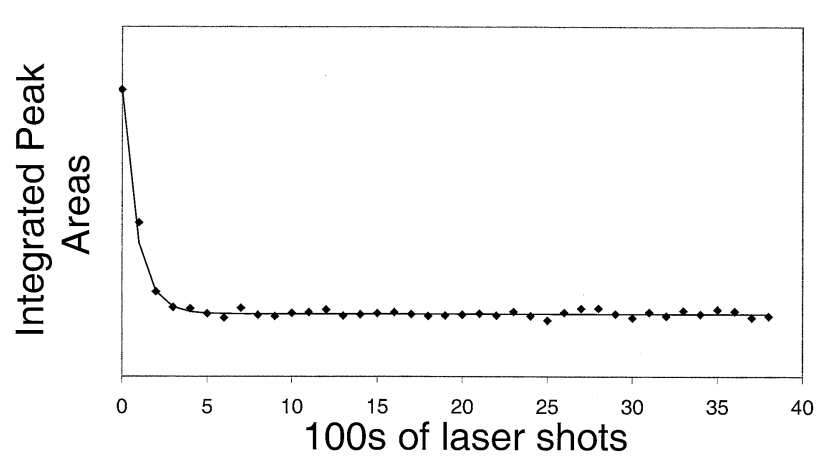

Figure 3. Integrated pyrene peak areas versus 100s of laser shots for a sample containing $10 \mu$ moles of pyrene/gram of charcoal. Each point represents the average of 100 mass spectra.

In order to determine the reproducibility of the standard samples, several trials were preformed in which one standard was exposed to several thousand laser shots; the result is provided in Figure 3 which is a plot of the magnitude (integrated pyrene peak areas) of the pyrene signal as a function of the number of laser shots for a sample containing $10 \mu \mathrm{mol}$ pyrene/gram charcoal. Each point on the graph represents the integrated peak area averaged over one hundred laser cycles/mass spectra. The data in Figure 3 indicate that there are likely two types of pyrene environments in the charcoal sample. For one adsorption site, the pyrene is weakly bound and it is desorption from this site that is primarily responsible for the pyrene signal during the first 500 laser shots. For the other type of adsorption site pyrene is bound more strongly and produces the relatively constant signal observed in the later tens of thousands of mass spectra. For the latter, the result that the pyrene signal is relatively stable for several thousand laser shots is practically useful, because it provides a stable source of analyte such that instrumental parameters and operating conditions can be optimized.

It is well known that the magnitude of the IR power plays an important role in the desorption process [14, $38,39]$, therefore the effect of IR intensity on the PAH signals was investigated. An example of this experiment is shown in Figure 4 for the case of phenanthrene

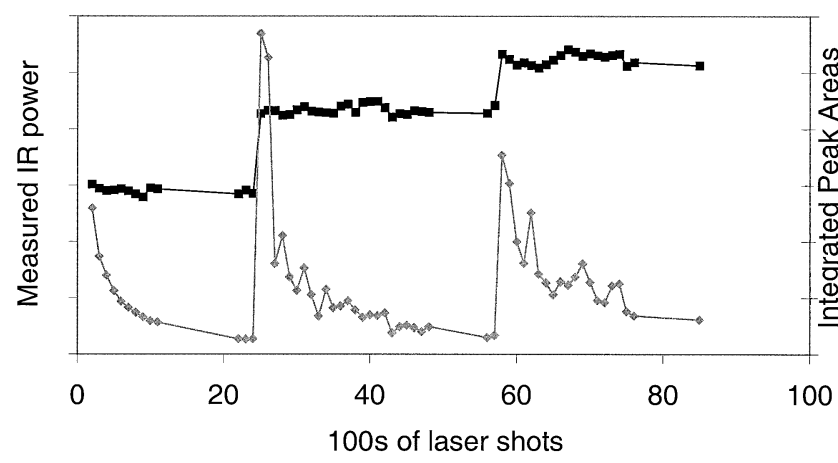

Figure 4. Integrated pyrene peak areas (filled diamond) and measured IR intensity (filled square) versus 100s of laser shots for a sample containing $10 \mu$ moles pyrene/gram of charcoal. Each point represents the average of 100 mass spectra. (integrated peak areas) at a concentration of $10 \mu \mathrm{mol}$ phenanthrene/gram of charcoal. Figure 4 shows an experiment where, like above, a sample was exposed to several thousand laser shots. For the first 2500 shots (IR laser power approximately $1.2 \times 10^{5} \mathrm{~W} / \mathrm{cm}^{2}$ ) the signal is observed to decrease and level off as before. At this point, the IR laser intensity was increased by 33\% (approximately $1.6 \times 10^{5} \mathrm{~W} / \mathrm{cm}^{2}$ laser power). As a result of this increase in IR power the phenanthrene signal increased sharply, and then began a downward trend as before. The same phenomenon was observed a third time when the laser power was increased yet again by $80 \%$ compared to the original (approximately $2.2 \times 10^{5} \mathrm{~W} / \mathrm{cm}^{2}$ laser power). These observations are consistent with bulk laser sampling theory [40-42]. Briefly, in order for a PAH to be desorbed from the surface of a bulk solid, the temperature must increase enough to make the process statistically probable. During the course of a $10 \mathrm{~ns}$ laser pulse, the laser induces very rapid heating in the solid. The rate of heating as well as the temporal and spatial properties of the temperature in a solid are affected by a number of factors including; the absorption coefficient of the material at that laser wavelength, the thermal conductivity of the sample, and the specific heat capacity of the material [14, 39]. Several excellent treatments of this heating phenomena [42-46] show that typically, the bulk sample will rise to a temperature that makes the desorption probable for only a few tens of nanoseconds during and after the laser pulse. These publications also show that, typically, when a greater IR power is applied to the sample, a higher temperature is achieved at a greater bulk depth. Therefore, we may conclude that in the case of a stepped IR profile, we are observing a sampling from greater and greater depths with each increase in IR power. The signals all quickly decay, as seen previously, because as the number of laser shots increases, the sampling area becomes depleted in weakly bound PAHs. Finally, in the third IR power step, the increase in phenanthrene signal is not as pronounced as the second step; this is most likely due to the fact that the PAHs must desorb from a further depth in order to be sampled.

The UV power is also well known to affect the type and magnitude of observed signals in two-laser mass spectrometry. As a result, a systematic study was undertaken to examine the effect of the UV power on the signal from several PAHs. Virtually identical results were observed for all of the five PAHs examined. Figure 5 is a plot of the UV power versus the integrated acenaphthene peak areas. The graph exhibits a linear relationship between signal amplitude and laser energy at energies below $80 \mu \mathrm{J}$, however, at higher energies the acenaphthene signal begins to level off (a line of best fit has been added to the data to aid the reader). At pulse energies higher than $100 \mu \mathrm{J}$, fragmentation products from the PAHs begin to appear in the mass spectra. This would indicate that at high photon fluxes, the neutrals are either absorbing three photons causing fragmenta- 


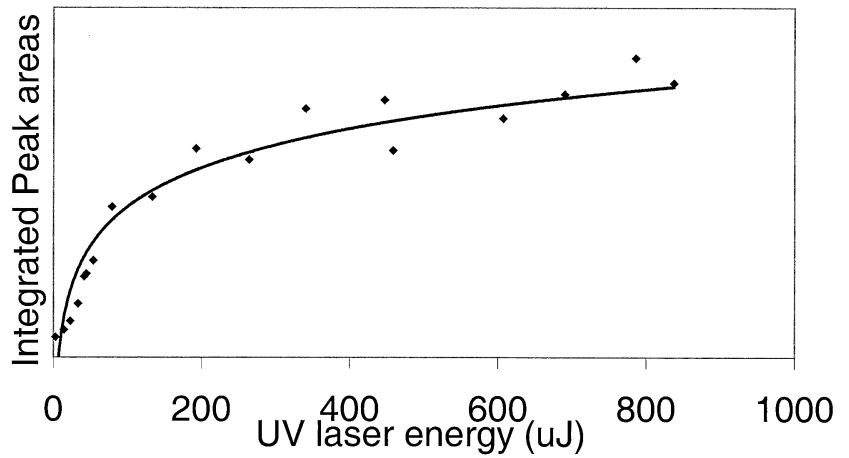

Figure 5. Integrated acenaphthene peak areas versus measured UV energy at a steady IR power for a sample containing $10 \mu$ moles of acenaphthene/gram of charcoal. Each point represents the average of 100 mass spectra.

tion, or ions formed previously are absorbing a photon to induce fragmentation. The relationship between the signal intensity and UV energy is similar to that observed by Zenobi's group [32] and is consistent with the theoretical treatment by Johnson and Otis [47]. Briefly, the results can be explained if one considers the processes involved in resonance enhanced two-photon ionization. If the absorption cross section at the excitation wavelength used is identical for both the ground state and the excited state of the molecule then one would expect to see a quadratic dependence of the signal on the UV energy. If, however, the absorption cross section at the excitation wavelength was much greater for one state than the other, a "rate determining step" would be observed, and the signal would become linear with UV intensity. This indeed is what was observed in the case of all five PAHs examined. It is impossible to know, for this experimental set-up, which absorption process is rate determining. The important result from this experiment is that it allows us to select the region around $\sim 40 \mu \mathrm{J} /$ pulse as the optimum $\mathrm{UV}$ laser energy for analytical determinations because the signal/energy ratio in this region is linear and thus is easily accounted for.

To be useful for analytical measurements, this methodology should provide quantitative capability. To evaluate this aspect, a series of standards of known concentration were prepared covering the concentration range 1 to $25 \mu \mathrm{mol}$ pyrene/gram of charcoal. The result is plotted in Figure 6. This plot exhibits a linear relationship between concentration and observed signal with an $\mathrm{R}^{2}$ value of 0.99 . The error bars are based on measuring five replicates of the same sample and are $+/-5 \%$. It should be noted, that this calibration curve demonstrates only the linear response of the system and not the absolute detection limits. This can be appreciated when one considers that the magnitude of the peak area is a function of both IR and UV laser energy as well as the concentration of the analyte and the nature of the matrix. As a result, the magnitude of the analyte

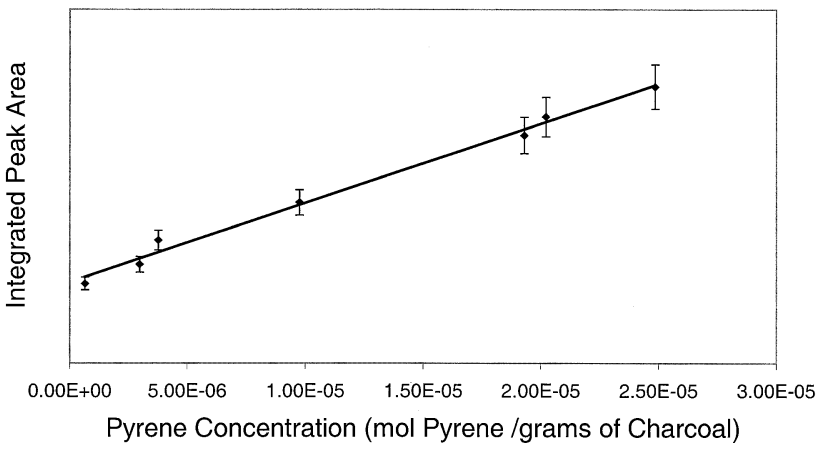

Figure 6. Integrated pyrene peak areas versus concentration of pyrene samples. Each point represents the average of 100 mass spectra.

signal can be significantly changed for a given concentration by simply increasing the IR and UV power. Calibration curves have been observed previously for the two-laser technique, however, most have used an internal standard to compensate for shot to shot fluctuations in the laser energy $[32,48]$. Regardless, a useable calibration curve was obtained in this experiment, which demonstrates that the technique is useful for, at least, semi-quantitative analysis. For precise quantitation the method of standard additions or an internal standard would, of course, produce the best results.

\section{Selective Ion Accumulation}

To demonstrate the ion traps unique capabilities, a series of experiments were performed to evaluate the possibility of selectively accumulating the results of several laser shots. External waveforms were used to isolate specific, low concentration species in the ion trap. These species have then been pre-concentrated in the gas phase by performing multiple laser shot/ SWIFT sequences while maintaining the RF voltage at a steady "trapping level". This procedure begins with desorption and ionization laser shots, followed by a short SWIFT pulse that is used to clear the trap of all species but the low concentration analyte. Successive laser shot/SWIFT pulse combinations are then used to build up a strong analyte population in the ion trap. Without the SWIFT pulses between laser shot combinations, all species in the trap would build up equally, and as a result the low intensity signal would be swamped.

As a demonstration of this method, a charcoal sample was prepared which contained five PAHs: Acenapthelene, phenanthrene, pyrene, benzo(a)pyrene, and coronene all at a concentration of approximately 25umol PAH/gram charcoal. A sixth PAH, chrysene, was also spiked on this sample, at a concentration of approximately $1 \mathrm{umol} \mathrm{PAH} /$ gram charcoal. An example of a mass spectrum of this sample collected in the normal manner is shown in the solid line in Figure 7. The chrysene signal $(228 \mathrm{~m} / \mathrm{z})$ is seen to be only slightly 


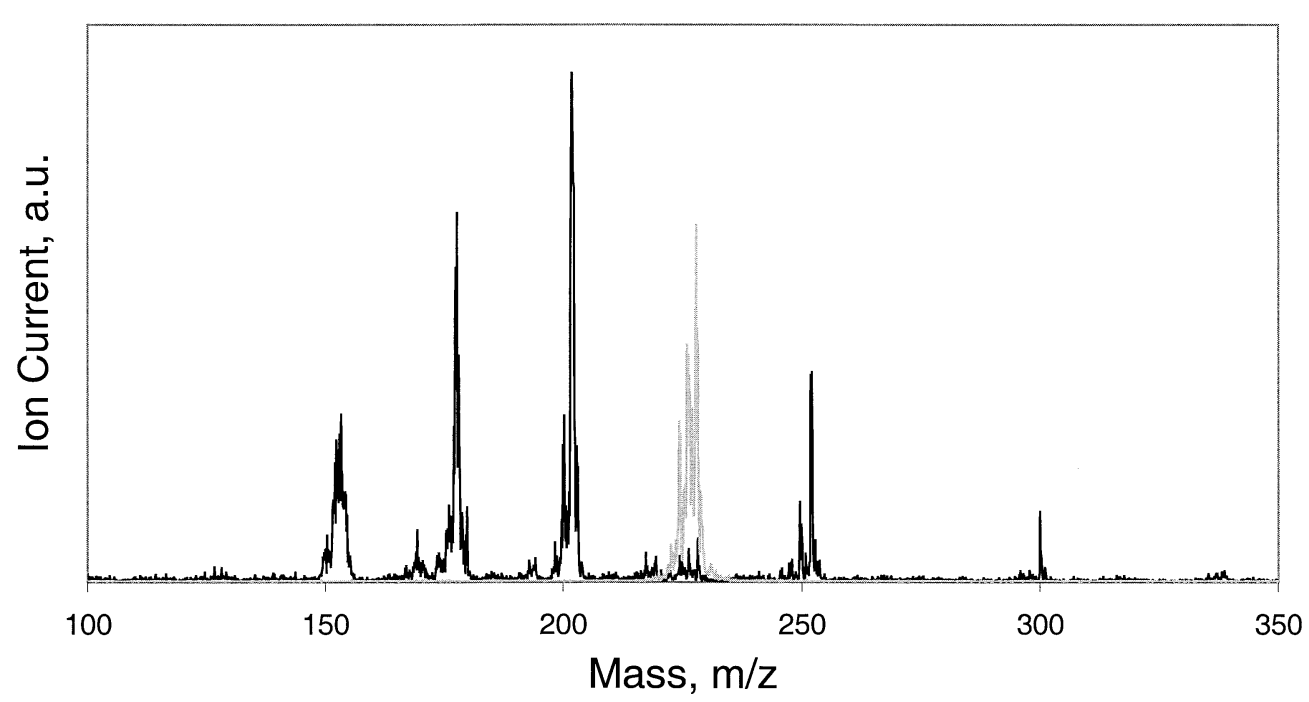

Figure 7. Two-laser mass spectrum of sample containing five PAHs (acenaphthene-154 Da, phenanthrene-178 Da, pyrene-202 Da, benzo(a)pyrene-252 Da, coronene-300 Da) all of equivalent concentration (25 $\mu$ moles of PAH/gram charcoal) and with chrysene-228 Da an order of magnitude lower in concentration ( $1 \mu$ moles of $\mathrm{PAH} /$ gram charcoal). (Dark line) without gas phase pre-concentration. (Light line) with gas phase pre-concentration.

above the base line in this spectrum. The gray line in Figure 7 shows a spectrum where seven laser shot/ SWIFT cycles were used to accumulate a large chrysene ion population. In this spectrum, the chrysene signal is now far above base line and easily quantifiable. This process was examined for several laser/SWIFT cycles. Figure 8 shows the average chrysene peak height (normalized relative to a single laser cycle) as a function of the number of cycles. This line has a slope of 0.9991 and a $R^{2}$ of 0.996 indicating that the build-up is very linear and that successive laser shots do not interfere with the trapped ions. This procedure could become analytically useful in situations where low concentration species are to be examined by MS/MS. Obviously, a signal build-up like this would be impossible in a TOF MS.

\section{SRM 1944 Analysis}

The final sets of experiments were aimed at determining whether or not real solid samples could be analyzed

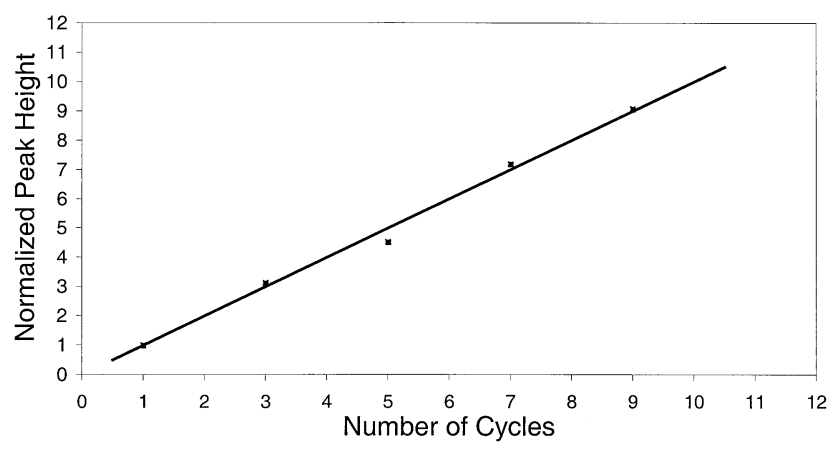

Figure 8. Integrated chrysene peak areas averaged over 100 laser shots and normalized with respect to one laser cycle versus number of laser/SWIFT cycles. with the proposed protocol. To this end, a standard reference material, SRM 1944 New York-New Jersey River water sediment, was examined in a manner identical to the determinations of the charcoal standards. The resulting spectrum produced with no sample pre-treatment is shown in Figure 9. This figure shows the uncorrected mass spectral data that results when a real sediment sample containing a series of PAHs along with several other classes of contaminants is analyzed. Table 1 shows a list of some of the PAH compounds certified to exist in this sample and their certified concentrations. It was beyond the scope of this study to quantify and confirm the concentrations of all the PAHs in this sample. Instead, this should simply be accepted as a proof of concept to stimulate further investigation. In the case of this experiment no real secondary layer of selectivity, beyond optical selectivity, is required in the analysis of the spectra; however, it would be a relatively trivial matter to perform SWIFT isolation on selected mass peaks for further daughter analysis and structural confirmation. This confirmation of mass selected peaks is impossible with most conventional two-laser systems because they all almost exclusively use TOF mass spectrometers for analysis. Unfortunately, the procedure as it currently exists, is incapable of discriminating between many PAH isomers. PAHs are one of a very small class of compounds for which MS/MS is poor at distinguishing between isomers because the isomers all have relatively similar fragmentation spectra. As a result, future work will focus on both demonstrating the MS/MS capability of the system (detecting drug molecules on biological matrices) and on $\mathrm{PAH}$ isomer discrimination using wavelength specific photo fragmentation. 


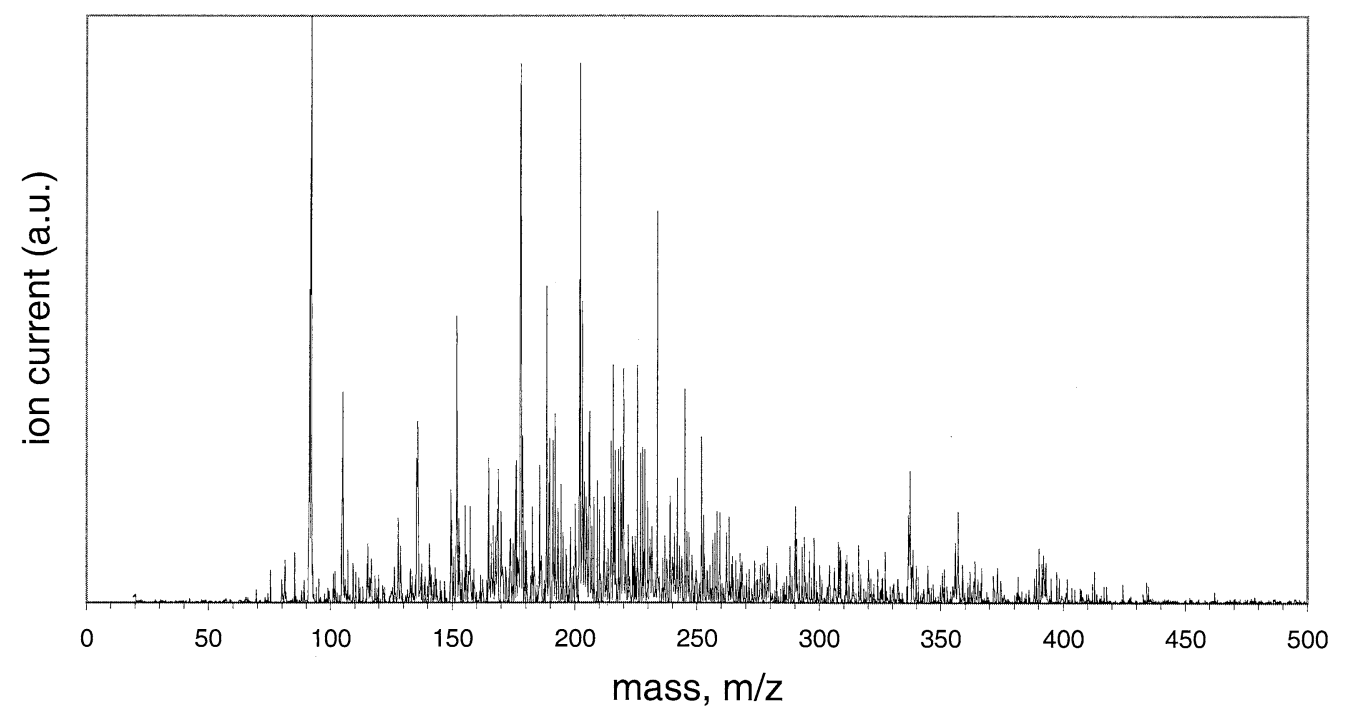

Figure 9. Two-laser mass spectrum of standard reference material 1944 New York/New Jersey River water sediment with no sample pre-treatment.

\section{Conclusions}

REMPI-ITMS can be effectively used to directly obtain mass spectra for PAHs in soils and similar materials. The REMPI method provides excellent selectivity for the PAHs coupled with a very high ionization efficiency. The ion trap was demonstrated to have many advantages over traditional TOF instruments with respect to two-laser analysis, however, a few problems with ion traps remain unavoidable. Ion traps currently have a lower mass resolution, accuracy, and range than reflectron TOF's. Consequently, future versions of this instrument will be modified to include an ion trap/reTOF hybrid. While the samples examined here were

Table 1. Select polycyclic aromatic hydrocarbons previously identified in standard reference material SRM-1944 New York/ New Jersey River water sediment and there previously certified concentrations

PAH's identified in SRM 1944a-New York-New Jersey River water sediment

\begin{tabular}{llc}
$\begin{array}{l}\text { Mass } \\
\text { (Da) }\end{array}$ & \multicolumn{1}{c}{ Component } & $\begin{array}{c}\text { Mass fraction } \\
(\mathrm{mg} / \mathrm{kg})\end{array}$ \\
\hline \hline 128 & Napthalene & 1.65 \\
154 & Acenapthelene & 0.57 \\
178 & Phenantherene & 5.27 \\
178 & Anthracene & 1.77 \\
202 & Fluorantherene & 8.92 \\
202 & Pyrene & 9.7 \\
228 & Chrysene & 4.86 \\
228 & Benz(a)anthracene & 4.72 \\
252 & Benz(x)flouranthene where x $=\mathrm{b}, \mathrm{j}, \mathrm{k}, \mathrm{a}$ & 9.04 \\
252 & Benzo(x)pyrene where x $=\mathrm{a}, \mathrm{e}$ & 7.58 \\
252 & Perylene & 1.17 \\
276 & Benzo(ghi)perylene & 2.84 \\
276 & Indeno(1,2,3-cd)pyrene & 2.78 \\
192 & Methylphenantherene & 7.88 \\
206 & Dimethylphenantherene & 11.94 \\
\hline
\end{tabular}

relatively high in concentration there is no reason to believe that by simply increasing the laser power or ionization time that sensitivity on par with other environmental mass spectrometric methods couldn't be achieved. In the future we will investigate further applications of this technique by expanding the scope of sample types (e.g., biological samples) and analytes (e.g., PCBs, drugs).

\section{References}

1. Cooks, R. G.; Glish G. L.; McLuckey S. A.; Kaiser R. E. Chem. Eng. News March 25, 1991; p 26.

2. March, R. E. Int. J. Mass Spec. Ion Processes 1992, 118/119, 71-135.

3. March, R. E. Int. J. Mass Spectrom. 2000, 200, 285-312.

4. Robb, D. R.; Blades, M. W. Int. J. Mass Spectrom. 1999, 190, 69-80.

5. Weil, C.; Nappi, M.; Cleven, C. D.; Wollnik, H.; Cooks, R. G. Rapid Commun. Mass Spectrom. 1996, 10, 742-750.

6. Ding, L.; Kawatoh, E.; Tanaka K.; Smith A. J.; Kumashiro S. Proceedings of SPIE International Symposium on Optical Science, Engineering and Instrumentation, Denver, Colorado, July, 1999, pp. 144-155.

7. Weyssenhoff, H. V.; Seltze, H. L.; Schlag, E. W. Z. Naturforsch, Teil A 1985, 40A, 674 .

8. Tembreull, R.; Lubman, D. M. Anal. Chem 1986, 58, 1299-1303.

9. Grotemeyer, J.; Walterm, K.; Boesl, U.; Schlag, E. W. Org. Mass Spectrom. 1986, 21, 645.

10. Anex, D. S.; de Vries, M. S.; Knebelkamp, A.; Bargon, J.; Wendt,, H. R.; Hunzinker, H. E. J. Mass Spectrom. Ion Processes 1994, 131, 319.

11. Dale, M. J.; Jones, A. C.; Pollard, S.; Langridge-Smith, P. R. R. Environ. Sci. Technol. 1993, 27, 1693-1695.

12. Dale, M.; Jones, A.; Langridge-Smith, P. R. R.; Costello, K. F.; Cummins, P. G. Anal. Chem. 1993, 65, 793-801.

13. Kornienko, O.; Tinka, J.; Wijesundara, B. J.; Hanley, L. Anal. Chem. 1998, 70, 1208-1213.

14. Cavanagh, R. R.; Buntin, S. A.; Richter, L. J.; King, D. S. Atmos. Mol. Phys. 1990, 24, 365-376. 
15. Winograd, N.; Baxter, J. P.; Kimock, F. M. Chem. Phys. Lett. 1982, 82, 581.

16. Zhan, Q.; Zenobi, R.; Buseck, P. R.; Teerman, S. Energy Fuels 1997, 11, 144-149.

17. Voumard, P.; Zhan, Q.; Zenobi, R. Chem. Phys. Lett. 1995, 239, 89-94.

18. Kornienko, O.; Ada, E.; Hanley, L. Anal. Chem. 1997, 69, $1536-1542$.

19. Menzic, C. A.; Potocki, B. B.; Santodonato, J. Environ. Sci. Technol. 1992, 26, 1278.

20. Clar, E. Polycyclic Hydrocarbons. Academic Press: London, 1964.

21. Giger, W.; Schaffner, C. Anal. Chem. 1978, 50, 243-249.

22. McDow, S. R.; Giger, W.; Burtscher, H.; Schmidt-Ott, A.; Siegmann, H. C. Atmos. Environ. 1990, 24a, 2911-2916.

23. Robbat, A.; Liu, T. Y.; Abraham, B. M. Anal. Chem. 1992, 64, 1477.

24. Mellone, A.; Smith, B. M.; Winefordner, J. D. Talanta 1990, 37, 111.

25. Niessner, R.; Robers, W.; Krupp, A.; Fresenius, J. Anal. Chem. 1991, 341, 207-213.

26. Pollard, S. J. T.; Hrudey, S. E.; Fuhr, B. J.; Alex, R. F.; Holloway, L. R.; Tosto, F. Environ. Sci. Technol. 1992, 26, 2528.

27. Day, R. J.; Unger, S. E.; Cooks, R. G. Anal. Chem. 1980, 52, 557A.

28. Duke, G. Org. Mass Spectrom. 1984, 19, 242.

29. Balasanmugam, K.; Viswanadham, S. K.; Hercules, D. M. Anal. Chem. 1986, 58, 1102.

30. Gillette, J. S.; Luthy, R. G.; Clement, S. J.; Zare, R. N. Environ. Sci. Technol. 1999, 33, 1185-1192.

31. Dale, M.; Jones, A.; Pollard, S.; Langridge-Smith, P. Analyst 1994, 119, 571-578.
32. Haefliger, O. P.; Zenobi, R. Anal. Chem 1998, 70, 2660-2665.

33. Zenobi, R.; Phillippoz, J. M.; Buseck, P. R.; Zare, R. N. Science 1989, 246, 1026.

34. McKay, D. S.; Gibson, E. K.; Thomas-Keprta, K. L.; Vali, H.; Romanek, C. S.; Clemett, S. J.; Chillier, X. D.; Maechling, C. R.; Zare, R. N. Science 1996, 273, 924-930.

35. Robb, D.; Blades, M. W. J. Am. Soc. Mass Spectrom. 1997, 8, 1203-1205.

36. de Vries, M. S. Rev. Anal. Chem. 2000, 19, 269-287.

37. Wright, S. J.; Dale, M. J. D.; Langridge-Smith, P. R. R.; Zhan, Q.; Zenobi, R. Anal. Chem. 1996, 68, 3585-3594.

38. Ready, J. F. Effects of High Power Laser Radiation. Academic Press: London, 1971, pp 67-124.

39. Cotter, R. J.; Tabet, J. C. Int. J. Mass Spectrom. Ion Phys. 1983, 53, 151-166.

40. Redhead, P. A. Vacuum 1962, 12, 203-211.

41. Cowin, J. P.; Auerbach, D. J.; Becker, C. A.; Wharton, L. Surf. Sci. 1978, 78, 545.

42. Philippoz, J. M.; Zenobi, R.; Zare, R. N. Chem. Phys. Lett. 1989, $158,12$.

43. Handschuh, M.; Nettesheim, S.; Zenobi, R. J. Chem. Phys. 1997, 107, 2603-2610.

44. Handschuh, M.; Nettesheim, S.; Zenobi, R. Chem. Phys. Lett. 1997, 275, 93-97.

45. Maechling, C. R.; Clemett, S. J.; Engelke, C. F.; Zare, R. N. J. Chem. Phys. 1996, 104, 8768-8776.

46. Nettesheim, S.; Zenobi, R. Chem. Phys. Lett. 1996, 255, 39-44.

47. Johnson, P. M.; Otis, C. E. Annu. Rev. Phys. Chem. 1981, 32, 139-157.

48. Zare, R. N.; Hahn, J. H.; Zenobi, R. Bull. Chem. Soc. Jpn. 1988, $61,87-92$. 